ARTICLE

\title{
Carrier-envelope phase-dependent high harmonic generation in the water window using few-cycle infrared pulses
}

Nobuhisa Ishii ${ }^{1}$, Keisuke Kaneshima ${ }^{1}$, Kenta Kitano ${ }^{1}$, Teruto Kanai ${ }^{1}$, Shuntaro Watanabe ${ }^{2}$ \& Jiro Itatani $^{1}$

High harmonic generation ( $\mathrm{HHG}$ ) using waveform-controlled, few-cycle pulses from Ti:sapphire lasers has opened emerging researches in strong-field and attosecond physics. However, the maximum photon energy of attosecond pulses via HHG remains limited to the extreme ultraviolet region. Long-wavelength light sources with carrier-envelope phase stabilization are promising to extend the photon energy of attosecond pulses into the soft X-ray region. Here we demonstrate carrier-envelope phase-dependent HHG in the water window using sub-two-cycle optical pulses at $1,600 \mathrm{~nm}$. Experimental and simulated results indicate the confinement of soft $\mathrm{X}$-ray emission in a single recombination event with a bandwidth of $75 \mathrm{eV}$ around the carbon $\mathrm{K}$ edge. Control of high harmonics by the waveform of few-cycle infrared pulses is a key milestone to generate soft $X$-ray attosecond pulses. We measure a dependence of half-cycle bursts on the gas pressure, which indicates subcycle deformation of the waveform of the infrared drive pulses in the HHG process.

\footnotetext{
${ }^{1}$ The Institute for Solid State Physics, The University of Tokyo, 5-1-5 Kashiwanoha, Kashiwa, Chiba 277-8581, Japan. ${ }^{2}$ Research Institute for Science and Technology, Tokyo University of Science, 2641 Yamasaki, Noda, Chiba 278-8510, Japan. Correspondence and requests for materials should be addressed to N.I. (email: ishii@issp.u-tokyo.ac.jp).
} 
W aveform-controlled, few-cycle optical pulses from Ti:sapphire lasers have opened the opportunity to explore electric-field-sensitive strong-field experiments and to generate attosecond pulses for spectroscopic applications ${ }^{1}$. However, the maximum photon energy of isolated attosecond pulses via high harmonic generation (HHG) using an 800 -nm electric field has been limited in the extreme ultraviolet (EUV; $<200 \mathrm{eV}$ ) because of the cutoff law in $\mathrm{HHG}^{2}$. To extend the spectral range of high harmonics (HHs) from the EUV to the soft X-ray region, long-wavelength light sources are promising candidates. This contradictory argument can be explained with a semi-classical three-step model ${ }^{2}$. The first step is tunnelling ionization at the peak of a strong electric field, which releases an electron wavepacket into the continuum from an atom. In the second step, the wavepacket is accelerated by the field and gains substantial kinetic energy. In the final step, the wavepacket is driven back to the vicinity of the parent ion by the reversed electric field and recombines with it, emitting a photon of energy equal to the sum of the kinetic energy and the ionization potential. The highest possible photon energy within this framework is called as the cutoff energy, and it is given by $E_{\text {cutoff }}=I_{\mathrm{p}}+3.17 U_{\mathrm{p}}$, where $I_{\mathrm{p}}$ is the ionization potential of a target atom, $U_{\mathrm{p}}[\mathrm{eV}]=9.3 \times 10^{-14} I\left(\mathrm{~W} \mathrm{~cm}^{-2}\right) \times(\lambda(\mu \mathrm{m}))^{2}$ is the ponderomotive energy, and $I$ and $\lambda$ are the laser intensity and wavelength, respectively. The maximum intensity applicable to a target medium is limited by the saturation of its ionization, which shows no dependence on the centre wavelength of the electric field and small dependence on its temporal duration. Thus, it is evident that the cutoff energy can be significantly extended using a long-wavelength electric field because of the square dependence of $U_{\mathrm{p}}$ on $\lambda$. Pioneering HHG experiments have demonstrated the usability of infrared pulses to extend the cutoffs ${ }^{3,4}$. Furthermore, recent experiments using multimillijoule pulses from infrared or mid-infrared optical parametric amplifiers (OPAs) have achieved drastic extension of the cutoff to the water window ${ }^{5}$ and even to more than $1 \mathrm{keV}$ (Popmintchev et al. ${ }^{6}$ ). These experiments have used relatively long femtosecond infrared pulses without controlling a carrierenvelope phase (CEP). As a result, the temporal resolution obtained with such HHs is generally limited by the envelope of the drive pulses and remains on a femtosecond time scale. Stabilization of the CEP of few-cycle pulses is essential to trigger the dynamics of a quantum system with subcycle accuracy and, therefore, with attosecond temporal resolution. Recent success in attosecond physics has exclusively owed to CEP-stabilized, fewcycle optical pulses at $\sim 800 \mathrm{~nm}$ based on Ti:sapphire laser technologies ${ }^{7-10}$

Progress in frequency conversion of optical pulses based on either broadband OPAs or optical parametric chirpedpulse amplifiers (OPCPAs) has realized the generation and amplification of few-cycle intense pulses in the infrared with CEP stabilization ${ }^{11-13}$. Such high-energy infrared light sources have been applied to the extension of the HH cutoffs ${ }^{14-16}$ up to $200 \mathrm{eV}$. However, a CEP dependence of soft X-ray harmonics, particularly beyond the carbon $K$ edge, has not been demonstrated.

We have developed an OPCPA using $\mathrm{BiB}_{3} \mathrm{O}_{6}$ (BIBO) crystals pumped by a Ti:sapphire laser ${ }^{17}$. BIBO-based OPAs or OPCPAs possess an extremely broadband gain spectrum, which extends nearly one octave $(1,200-2,200 \mathrm{~nm})$ under a collinear phasematching condition at degeneracy when pump lasers at $800 \mathrm{~nm}$ are used ${ }^{18,19}$. In a BIBO-OPCPA, few-cycle, intense infrared pulses can be directly generated from the amplifier without nonlinear spectral broadening. The output pulses from our BIBO-OPCPA have extremely good shot-to-shot stability both in the output energy and the CEP: $0.85 \%$ (root mean square; r.m.s.) and $160 \mathrm{mrad}$ (r.m.s.), respectively ${ }^{17}$. The long-term drifts of the output energy and the CEP are also measured to be better than $2 \%$ and $100 \mathrm{mrad}$ over $24 \mathrm{~h}$, respectively. In this work, we generate HHs from neon, which exhibit spectral extension to the water window with a clear dependence on the CEP of the sub-two-cycle infrared pulses from the OPCPA. The observed CEP dependence is well reproduced by simulation results based on the strong-field approximation (SFA) ${ }^{20}$ and shows a clear separation of the spectra of half-cycle soft X-ray bursts. Our experimental and simulated results indicate the confinement of soft X-ray emission in a single recombination event, which has a bandwidth of $75 \mathrm{eV}$ around the carbon $K$ a

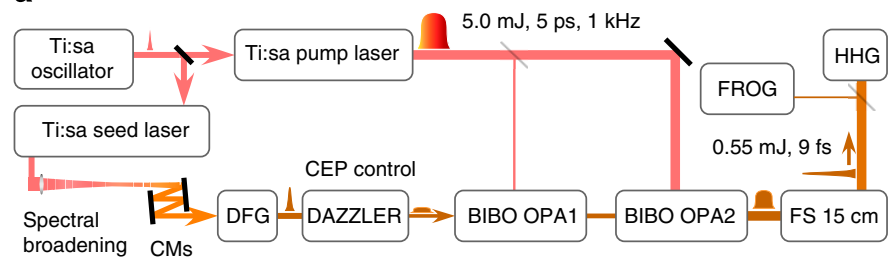

C

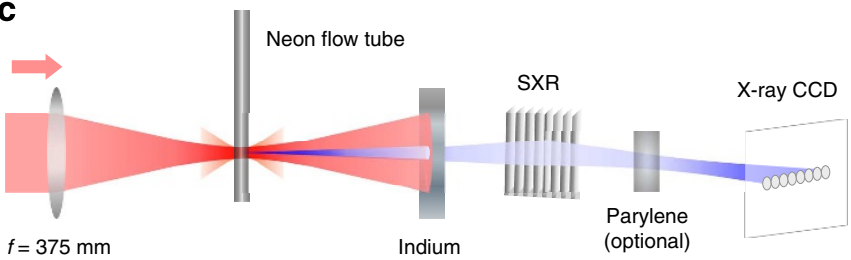

b
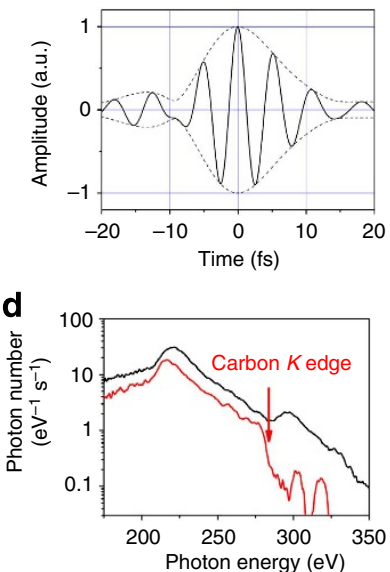

Figure 1 | Experimental set-up. (a) Schematic of the IR OPCPA system. Difference frequency generation (DFG) crystal for CEP-stabilized seed pulses; DAZZLER acousto-optic programmable dispersive filter (Dazzler, FASTLITE); BIBO OPA 1 and OPA2, first and second BIBO OPA stages, respectively; FS, 150-mm-long glass block made of synthetic fused silica; FROG, frequency-resolved optical gating apparatus; HHG, high harmonic generation set-up. (b) Electric field of the 1,600-nm, 9.0-fs optical pulses with a cos-like waveform (solid line) and its envelope (dashed lines). (c) Set-up for the generation of soft X-ray HHs detected by the X-ray charge-coupled device camera after the soft X-ray spectrometer (SXR). A 150-nm-thick indium filter is inserted to block the fundamental beam and low-order harmonics below $140 \mathrm{eV}$. (d) HH spectra recorded with (red line) and without (black line) two 200-nm-thick parylene filters. The sharp intensity drop indicates the absorption of the carbon $\mathrm{K}$ edge at $284 \mathrm{eV}$ by the parylene filters. 
edge. We also observe that the cutoffs of half-cycle bursts respond differently to the backing pressure of the neon gas, which reveals subcycle nonlinear deformation of the few-cycle infrared pulses in the HHG process.

\section{Results}

HHG in the water window. We used optical pulses at a wavelength of $1,600 \mathrm{~nm}$ and a repetition rate of $1 \mathrm{kHz}$ from the infrared OPCPA (Fig. 1a) for HHG experiments. This light source is capable of delivering $9.0 \mathrm{fs}, 550 \mu \mathrm{J}$, CEP-stabilized optical pulses. Figure $1 \mathrm{~b}$ shows an example of the cos-like waveform of the $9.0 \mathrm{fs}$ infrared pulses, which were characterized by frequencyresolved optical gating. The OPCPA output beam was focused by a spherical mirror with a focal length of $375 \mathrm{~mm}$ into a $1.4-\mathrm{mm}$ thick gas cell filled with neon set in a vacuum chamber (Fig. 1c). The backing pressure of the cell was varied up to $1.4 \mathrm{~atm}$. The pulse energy of the infrared beam was adjusted to be $380 \mu \mathrm{J}$ using a diaphragm with a 20 -mm-diameter aperture for all the measurements in this work. The fundamental beam and low-order harmonics below $\sim 140 \mathrm{eV}$ were blocked by a 150 -nm-thick indium filter, which was placed in front of a soft X-ray spectrometer (SXR-II-1, Hettrick Scientific). Spectrally resolved HHs were detected by a back-illuminated X-ray charge-coupled device camera (PIXIS-XO-2048B, Princeton Instruments). The exposure time of the camera was set to be $10 \mathrm{~s}$. The red and black lines of Fig. 1d plot $\mathrm{HH}$ spectra that were recorded with and without two additional 200-nm-thick parylene filters, respectively. The sharp intensity drop at $284 \mathrm{eV}$ of the red line is due to the absorption of the carbon $K$ edge. Intensity distributions of harmonic spectra are plotted by the photon flux (photon number per eV per second) in front of the camera after intensity calibration.

CEP-dependent half-cycle cutoffs. A relative CEP, $\Delta \varphi_{\mathrm{CEP}}$, of the infrared pulses was scanned by an acousto-optic programmable dispersive filter (Dazzler, HR45-1200-2200, FASTLITE). Note that $\Delta \varphi_{\mathrm{CEP}}$ is a relative value, of which the phase offset was adjusted to match experimental data with simulated results. Figure 2a shows $\mathrm{HH}$ spectra that were recorded at four CEPs in steps of $0.3 \pi \mathrm{rad}$. We found that one or two peaks in the $\mathrm{HH}$ spectra were drastically shifted by the change of the CEP at a rate of $75 \mathrm{eV}(\pi \mathrm{rad})^{-1}$. These peaks are attributed to be half-cycle cutoffs (HCOs) that originate from several electron trajectories allowed in a few-cycle pulse-driven HHG process ${ }^{21}$. The spectral density of each dipole emission increases near the cutoff, which results in the spectral peaks. Such spectral structures have been observed both theoretically ${ }^{2}$ and experimentally ${ }^{21}$. To understand the behaviour of the observed HCOs, we simulate the spectra of half-cycle bursts using the SFA. We consider an HHG process with two CEPs of a sub-two-cycle pulse as shown in Fig. 2c,d. These figures depict electron trajectories that are mainly allowed under the cos- and sin-like waveforms, respectively. Figure $2 \mathrm{~b}$ represents the spectral distributions of electric dipoles as a result of the electron trajectories that are shown in Fig. 2c,d. Parameters used in the simulations are listed in the caption of Fig. 2. Shifting the CEP of a few-cycle field significantly changes the amplitude of each half cycle. Therefore, the cutoff energy of soft X-ray emission from each trajectory exhibits a dependence on the CEP. The sum of the spectra of the individual bursts in Fig. $2 b$ well reproduces the experimental $\mathrm{HH}$ spectra in Fig. 2a. The experimental and the simulated $\mathrm{HH}$ spectra are quantitatively consistent in (i) the positions of the HCOs, (ii) the amount of the energy shift of the HCOs with respect to the CEP, and (iii) the intensity ratio between the two HCOs. The highest $\mathrm{HCO}$ reaches to $\sim 325 \mathrm{eV}$ as shown by the green line in Fig. 2a. This cutoff energy is consistent with previous works ${ }^{6,22}$.
We measured a collection of CEP-dependent $\mathrm{HH}$ spectra (Fig. 3a) that were recorded in steps of $0.1 \pi \mathrm{rad}$. The backing pressure of neon was set at $0.6 \mathrm{~atm}$. The $\mathrm{HH}$ spectra were reproduced every $\pi \mathrm{rad}$ of the CEP. This observation is a consequence of the HHG process because a CEP shift by $\pi \mathrm{rad}$ spatially inverts the electric field of drive pulses, which does not affect the spectral intensity of HHs. The SFA-based simulation in Fig. $3 b$ has been conducted to reproduce the observed CEP dependence. The best match to the experimental data is found by assuming a pulse duration of $10 \mathrm{fs}$ (FWHM of intensity), a peak intensity of $3.8 \times 10^{14} \mathrm{~W} \mathrm{~cm}^{-2}$ and a centre wavelength of $1,600 \mathrm{~nm}$. Here the experimental CEP values have an ambiguity of $\pi \mathrm{rad}$. The spectra of calculated dipoles contain modulated structures from interference among the two emissions from the short and long electron trajectories in a single recombination event. The emission from the latter is usually suppressed under appropriate phase-matching conditions ${ }^{23}$. The soft X-ray continuum generated in a single recombination event when $\Delta \varphi_{\mathrm{CEP}}=-0.3 \pi \mathrm{rad}$ as shown in Figs $2 \mathrm{a}$ and $3 \mathrm{a}$ has the maximum bandwidth of $\sim 75 \mathrm{eV}$ around the carbon $K$ edge. When higher laser intensity was applied onto the gas target, peak structures and CEP dependence in the $\mathrm{HH}$ spectra disappeared. This behaviour is most probably due to heavy ionization and associated plasma effects, which degrade the phase coherence of generated HHs. Such observation for $\mathrm{HH}$ spectra around $180 \mathrm{eV}$ has been demonstrated using near-infrared drive pulses at $800 \mathrm{~nm}$ (Ishii et al. ${ }^{24}$ ).

Pressure dependence of HCOs. In the next experiment, we investigated the dependence of the HCOs on the target gas pressure. This experiment was carried out in the same run with the equivalent parameters as those used in the previous measurement. The peak power of the infrared pulses was $38 \mathrm{GW}$, which is approximately two times more than the critical power of $21 \mathrm{GW}$ for self-focusing when the pressure of neon is set at $1.0 \mathrm{~atm}$ (Börzsönyi et al. ${ }^{25}$ ). Figure $4 \mathrm{a}$ shows the CEP dependence of soft X-ray spectra measured at a backing pressure of $1.0 \mathrm{~atm}$. By increasing the backing pressure from 0.6 to $1.0 \mathrm{~atm}$, on-axis intensities of HHs below $280 \mathrm{eV}$ increased by nearly one order of magnitude. The conversion efficiency from the infrared pulse to the soft X-ray continuum in the photon energy range between 150 and $300 \mathrm{eV}$ is calculated to be on the order of $10^{-9}$ after the spectrometer, which is consistent with HHG experiments that use $800 \mathrm{~nm}$ drive pulses by taking the wavelength scaling of $\lambda^{-(5-6)}$ into account ${ }^{26-28}$. The CEP-dependent $\mathrm{HH}$ spectra at $1.0 \mathrm{~atm}$ show a similarity to that observed with a backing pressure of $0.6 \mathrm{~atm}$. However, the spectral widths of the half-cycle bursts were broadened and their locations were shifted horizontally by $-(0.3-0.5) \pi \mathrm{rad}$ depending on the photon energies. We presume that such spectral dependences of the peak shift are caused by subcycle deformation of the waveform of the infrared pulses.

We scanned the backing pressure from 0.2 to $1.4 \mathrm{~atm}$ with a fixed CEP $\left(\Delta \varphi_{\mathrm{CEP}}=-0.3 \pi \mathrm{rad}\right)$ to examine responses of the HCOs. The pressure at the interaction region is estimated to be on the same order as the backing pressure of the gas cell because of low conductance through two tiny holes (diameter $<100 \mu \mathrm{m}$ ), which were drilled by the infrared beam. Figure $4 \mathrm{~b}$ plots the pressure dependence of the HCOs. By increasing the pressure from 0.2 to $0.4 \mathrm{~atm}$, the on-axis intensity of $\mathrm{HHs}$ rapidly increased, which indicates the onset of self-guiding of the infrared pulses $^{6}$. The HH spectrum at $0.6 \mathrm{~atm}$ in Fig. $4 \mathrm{~b}$ contains two HCOs located at $\sim 180$ and $280 \mathrm{eV}$. These two HCOs were shifted downwards with different slopes when the gas pressure was increased. The lower-energy peak downshifts with a slope of 

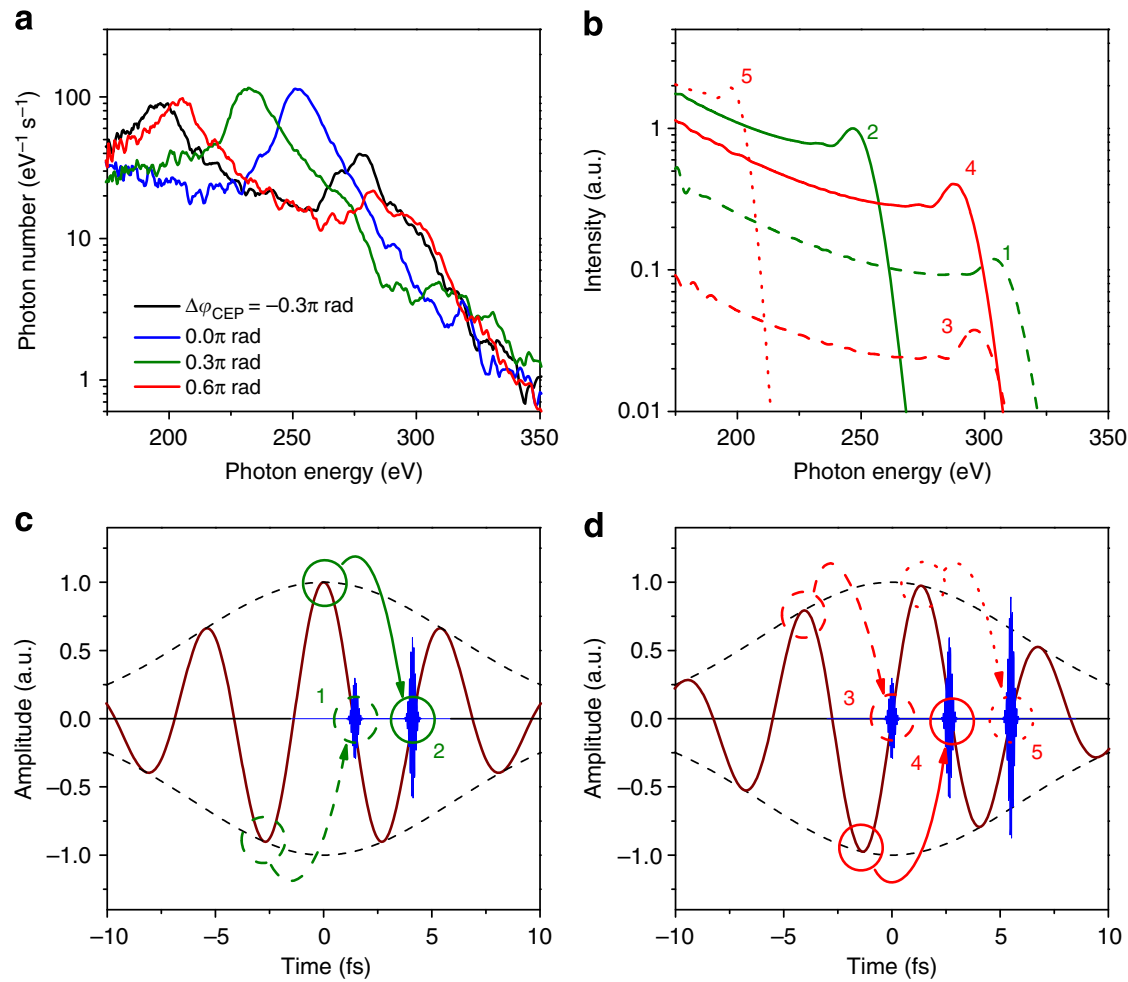

Figure 2 | CEP-dependent HCOs associated with the electron trajectories in a sub-two-cycle pulse. (a) Four HH spectra recorded at $\Delta \varphi_{\mathrm{CEPS}}$ in steps of $0.3 \pi$ rad. The spectra contain one or two peaks, which are attributed to the HCOs of harmonic emissions from two major electron trajectories. (b) Spectral distributions of soft X-ray emissions, which are calculated by an SFA-based simulation assuming 10-fs optical pulses at 1,600 nm with an intensity of $3.8 \times 10^{14} \mathrm{~W} \mathrm{~cm}^{-2}$. Each burst is filtered out by a temporal gate to select dipole emission contributed from the short trajectory. The duration of the gate was set to be approximately one-third of the oscillation period of a 1,600-nm field ( $\sim 1.8 \mathrm{fs}$ ). The green dashed and solid curves plot the spectral distributions of dipole emissions under the cos-like waveform, which result from the electron trajectories represented by the dashed and solid curves in Fig. 2c, respectively. The red dashed, solid and dotted curves plot the spectral distributions of dipole emissions under the sin-like waveform, which result from the electron trajectories represented by the dashed, solid and dotted curves in Fig. 2d, respectively. (c,d) Dominant electron trajectories under the cos-like and sin-like waveforms, respectively. The spectral distributions of electric dipoles are shown in Fig. $2 b$. The numbers and the colours associated with the electron trajectories are matched with Fig. $2 \mathrm{~b}$.
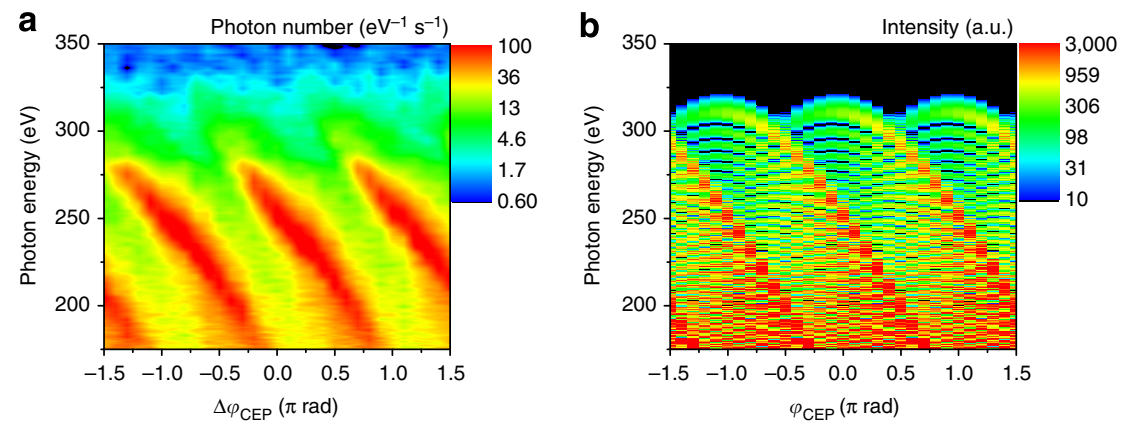

Figure 3 | CEP-dependent HH spectra. (a) Experimentally obtained HH spectra recorded at $\Delta \varphi_{\text {CEPS }}$ in steps of $0.1 \pi$ rad and at a backing pressure of $0.6 \mathrm{~atm}$. (b) Simulated $\mathrm{HH}$ spectra assuming $10 \mathrm{fs}$ optical pulses at $1,600 \mathrm{~nm}$ with an intensity of $3.8 \times 10^{14} \mathrm{~W} \mathrm{~cm}^{-2}$.

$-280 \mathrm{eV} \mathrm{atm}^{-1}$ (indicated by the black dashed line in Fig. 4b), whereas the higher-energy peak downshifts with a slope of $-130 \mathrm{eV} \mathrm{atm}^{-1}$ (the black solid line in Fig. 4b). This fact also supports the above-mentioned mechanism of waveform deformation induced by cycle-dependent phase modulation by nonlinear processes such as self-phase modulation, self-focusing and plasma effects. From these dependences, we can obtain information about cycle-dependent nonlinear interactions between the driver electric field and a gaseous medium.
Subcycle deformation of a driver laser field has been theoretically investigated ${ }^{29-31}$, and also discussed to explain the spectral shift of $\mathrm{HH}$ combs ${ }^{32-34}$. In our case, a two-dimensional plot can be used to reveal a pressure dependence of HCOs originated from consecutive electron trajectories as clear evidence of waveform deformation. The CEP dependences in Figs $3 \mathrm{a}$ and 4a also indicate that pressure-induced deformation upshifts the instantaneous frequency of the subcycle waveform. Such frequency upshift causes the reduction of the HCOs and the 

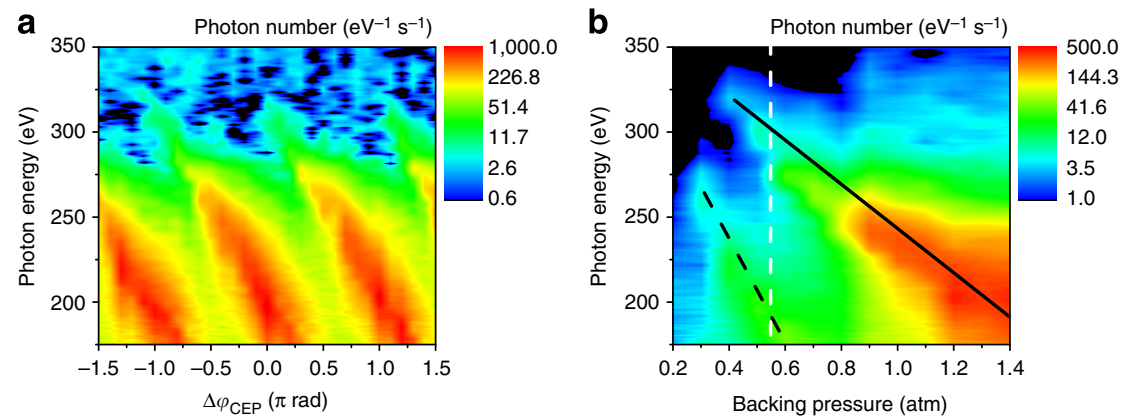

Figure 4 I CEP and pressure dependences of HH spectra. (a) Experimentally obtained HH spectra recorded at $\Delta \varphi_{\mathrm{CEPS}}$ in steps of $0.1 \pi$ rad and at a backing pressure of $1.0 \mathrm{~atm}$. (b) Pressure dependence of $\mathrm{HH}$ spectra. The black solid and dashed lines are drawn along the spectral peaks that originate from different HCOs. The white dashed line is drawn at the gas pressure of the self-focusing threshold $(0.55 \mathrm{~atm})$ when the critical power is identical to the peak power of the infrared pulses (38 GW).

increase of the slope that traces the spectral peaks at the HCOs in Fig. 4a compared with Fig. 3a.

\section{Discussion}

A CEP dependence of HHs using few-cycle Ti:sapphire lasers at $800 \mathrm{~nm}$ has usually been observed as spectral transition around the cutoff of the HHs from modulated structures to the appearance of a continuum in the $\mathrm{EUV}^{7}$. This method must spectrally resolve adjacent harmonic combs that are separated by twice the photon energy of Ti:sapphire laser pulses. For 1,600 nm drive pulses, this method requires a spectral resolution of a fraction of $1.5 \mathrm{eV}$ around $300 \mathrm{eV}$, which is difficult to achieve with a compact soft $\mathrm{X}$-ray spectrometer. Recent observation of CEP dependences using HCOs in the EUV has been used to experimentally determine the CEP of few-cycle Ti:sapphire laser pulses ${ }^{21}$. In this work, we have shown the dependence of the HCOs on the CEP of the few-cycle infrared pulses to determine the CEP value of the drive pulses. Our experimental and simulated results indicate, at a certain CEP value, the confinement of soft X-ray emission into a single recombination event near the cutoff, which has a $75-\mathrm{eV}$-wide bandwidth around the carbon $K$ edge. A wide continuum generated by a long-wavelength driver is more advantageous than that generated by Ti:sapphire lasers for potential generation of shorter attosecond pulses. To date, researchers have attained the shortest attosecond pulses with a sub-two-cycle temporal duration of 67 as in the $\mathrm{EUV}^{35}$. Further shortening of attosecond pulses requires longer-wavelength drivers to upshift their frequency to the soft X-ray region.

In conclusion, we applied the infrared light source based on the $\mathrm{BIBO}-\mathrm{OPCPA}$ scheme to generate $\mathrm{HH}$ in the water window that exhibit the clear dependence on the CEP of the sub-two-cycle infrared pulses. The highest $\mathrm{HCO}$ reaches $325 \mathrm{eV}$, which is well beyond the carbon $K$ edge. The experimental observations such as the spectral structures and CEP dependences of the half-cycle bursts are well reproduced by the SFA-based simulated results assuming $10 \mathrm{fs}, 1,600 \mathrm{~nm}$ optical pulses with an intensity of $3.8 \times 10^{14} \mathrm{~W} \mathrm{~cm}^{-2}$. The $\mathrm{HH}$ spectra consist of a few peaks located at different spectral positions; these peaks are attributed to the HCOs that originate from electron trajectories allowed in the few-cycle pulse-driven HHG process. The experimental and simulated results indicate the confinement of soft X-ray emission in a single recombination event, which has a bandwidth of $\sim 75 \mathrm{eV}$ around the carbon $K$ edge. This work shows the general capability of CEP-stable few-cycle intense infrared pulses towards generation of isolated coherent soft X-ray pulses and their applications to ultrafast soft X-ray spectroscopy with a temporal resolution from attoseconds to few femtoseconds.

\section{Method}

Infrared OPCPA system. Here we describe the details of the infrared OPCPA system. Readers are also referred to elsewhere ${ }^{18}$. A schematic of the OPCPA is presented in Fig. 1. The output pulses from a Ti:sapphire oscillator seeded two Ti:sapphire chirped-pulse amplification (CPA) systems where one system was used to produce infrared seed pulses, and the other to pump BIBO-OPAs. Femtosecond output pulses from one of the two CPA systems is focused into a 1.6-bar krypton gas cell for spectral broadening via filamentation. Infrared seed pulses were produced by difference frequency generation between the blue and red spectral components of the broadened spectrum. The generated seed pulses, which carry a stabilized CEP owing to the difference frequency generation process, were temporally stretched to about $4 \mathrm{ps}$ in the acousto-optic programmable dispersive filter. We obtained $5 \mathrm{~mJ}, 5 \mathrm{ps}$ OPA pump pulses at $1 \mathrm{kHz}$ and $805 \mathrm{~nm}$ from the other CPA system. The infrared seed pulses were amplified in two OPA stages, which consisted of 4-mm-thick and 6-mm-thick BIBO crystals both cut at $\theta=11.4^{\circ}$ for type I interaction in the $x z$ plane, respectively. The output energy after the first stage was typically $10 \mu \mathrm{J}$ using 0.2 -mJ pump pulses. We achieved an output energy of $550 \mu \mathrm{J}$ after the second OPA with a conversion efficiency of $12 \%$. We obtained nearly an octave gain bandwidth from 1,200 to 2,200 nm. Shot-to-shot energy fluctuations of the output pulses were measured to be $0.85 \%$ (r.m.s.). The amplified pulses were then compressed in a 150-mm-long glass block made of synthetic fused silica (SK1310, Ohara quartz). The compressed pulses were characterized by a third-harmonic generation-based frequency-resolved optical gating apparatus to have a duration of $9.0 \mathrm{fs}$ (FWHM), which contains a 1.7-cycle electric field at $1,600 \mathrm{~nm}$. The CEP stability of the amplified pulses was measured in a single-shot $f$-to- $2 f$ interferometer to be $160 \mathrm{mrad}$.

HHG chamber and spectrometer. The output beam from the OPCPA was focused into a 1.4-mm-thick gas cell filled with neon in the vacuum chamber (Fig. 1c). The backing pressure of the cell was limited up to $1.4 \mathrm{~atm}$ by the capacity of a turbomolecular pump (STP-A803C, Edwards) attached to the chamber. The HHG chamber was maintained at $2.8 \times 10^{-2} \mathrm{~Pa}$ when the backing pressure was set at $0.6 \mathrm{~atm}$, and the flow rate of neon was $40 \mathrm{sccm}$. Generated harmonics were spectrally dispersed by the soft X-ray spectrometer. This spectrometer images the source point of HHs to the charge-coupled device camera while HHs are spectrally resolved in one dimension. The spectrometer has been calibrated using parylene and boron filters to identify the $K$ edges of carbon at $284 \mathrm{eV}$ and boron at $188 \mathrm{eV}$, respectively.

\section{References}

1. Corkum, P. B. \& Krausz, F. Attosecond science. Nat. Phys. 3, 381-387 (2007)

2. Corkum, P. B. Plasma perspective on strong field multiphoton ionization. Phys. Rev. Lett. 71, 1994-1997 (1993).

3. Shan, B. \& Chang, Z. Dramatic extension of the high-order harmonic cutoff by using a long-wavelength driving field. Phys. Rev. A 65, 011804(R) (2001).

4. Colosimo, P. et al. Scaling strong-field interactions towards the classical limit Nat. Phys. 4, 386-389 (2008).

5. Takahashi, E. J., Kanai, T., Ishikawa, K. L., Nabekawa, Y. \& Midorikawa, K. Coherent water window $\mathrm{X}$ ray by phase-matched high-order harmonic generation in neutral media. Phys. Rev. Lett. 101, 253901 (2008).

6. Popmintchev, T. et al. Bright coherent ultrahigh harmonics in the keV X-ray regime from mid-infrared femtosecond lasers. Science 336, 1287-1291 (2012)

7. Baltuška, A. et al. Attosecond control of electronic processes by intense light fields. Nature 421, 611-615 (2003).

8. Kienberger, R. et al. Atomic transient recorder. Nature 427, 817-821 (2004).

9. Sansone, G. et al. Isolated single-cycle attosecond pulses. Science 314, 443-446 (2006). 
10. Wirth, A. et al. Synthesized light transients. Science 334, 195-200 (2011).

11. Fuji, T. et al. Parametric amplification of few-cycle carrier-envelope phasestable pulses at $2.1 \mu \mathrm{m}$. Opt. Lett. 31, 1103-1105 (2006).

12. Vozzi, C. et al. Millijoule-level phase-stabilized few-optical-cycle infrared parametric source. Opt. Lett. 32, 2957-2959 (2007).

13. Mücke, O. D. et al. Self-compression of millijoule $1.5 \mu \mathrm{m}$ pulses. Opt. Lett. 34, 2498-2500 (2009).

14. Schmidt, B. E. et al. High harmonic generation with long-wavelength few-cycle laser pulses. J. Phys. B: At. Mol. Opt. Phys. 45, 074008 (2012).

15. Gong, C. et al. Phase-matching mechanism for high-photon-energy harmonic of a long trajectory driven by a midinfrared laser. Phy. Rev. A 85, 033410 (2012)

16. Hong, K.-H. et al. High-order harmonic generation in $\mathrm{Xe}, \mathrm{Kr}$, and $\mathrm{Ar}$ driven by a 2.1- $\mu \mathrm{m}$ source: high-order harmonic spectroscopy under macroscopic effects. Phys. Rev. A 86, 043412 (2012).

17. Ishii, N. et al. Sub-two-cycle, carrier-envelope phase-stable, intense optical pulses at $1.6 \mu \mathrm{m}$ from a $\mathrm{BiB}_{3} \mathrm{O}_{6}$ optical parametric chirped-pulse amplifier. Opt. Lett. 37, 4182-4184 (2012).

18. Nikolov, I. et al. Ultrabroadband continuum amplification in the near infrared using $\mathrm{BiB}_{3} \mathrm{O}_{6}$ nonlinear crystals pumped at $800 \mathrm{~nm}$. Opt. Lett. 32, 3342-3344 (2007).

19. Ishii, N., Kitano, K., Kanai, T., Watanabe, S. \& Itatani, J. Carrier-envelopephase-preserving, octave-spanning optical parametric amplification in the infrared based on $\mathrm{BiB}_{3} \mathrm{O}_{6}$ pumped by $800 \mathrm{~nm}$ femtosecond laser pulses. Appl. Phys. Express. 4, 022701 (2011).

20. Lewenstein, M., Balcou, P. h., Ivanov, M. Y. u., L’Huillier, A. \& Corkum, P. B. Theory of high-harmonic generation by low-frequency laser fields. Phys. Rev. A 49, 2117-2132 (1994).

21. Haworth, C. A. et al. Half-cycle cutoffs in harmonic spectra and robust carrierenvelope phase retrieval. Nat. Phys. 3, 52-57 (2007).

22. Popmintchev, T. et al. Phase matching of high harmonic generation in the soft and hard X-ray regions of the spectrum. PNAS 106, 10516-10521 (2009).

23. Salières, P., L'Huillier, A. \& Lewenstein, M. Coherence control of high-order harmonics. Phys. Rev. Lett. 74, 3776-3779 (1995).

24. Ishii, N. et al. Generation of soft X-ray and water window harmonics using a few-cycle, phase-locked, optical parametric chirped-pulse amplifier. Opt. Lett. 37, 97-99 (2012).

25. Börzsönyi, Á., Heiner, Z., Kovács, A. P., Kalashnikov, M. P. \& Osvay, K. Measurement of pressure dependent nonlinear refractive index of inert gases. Opt. Express. 18, 25847-25854 (2010).

26. Gordon, A. \& Kärtner, F. X. Scaling of keV HHG photon yield with drive wavelength. Opt. Express. 13, 2941-2947 (2005).

27. Tate, J. et al. Scaling of wave-packet dynamics in an intense midinfrared field. Phys. Rev. Lett. 98, 013901 (2007).

28. Shiner, A. D. et al. Wavelength scaling of high harmonic generation efficiency. Phys. Rev. Lett. 103, 073903 (2009).

29. Geissler, M. et al. Light propagation in field-ionizing media: extreme nonlinear optics. Phys. Rev. Lett. 83, 2930-2933 (1999).
30. Christov, I. P. Propagation of ultrashort pulses in gaseous medium: breakdown of the quasistatic approximation. Opt. Express. 6, 34-39 (2000).

31. Yakovlev, V. S., Ivanov, M. \& Krausz, F. Enhanced phase-matching for generation of soft $\mathrm{X}$-ray harmonics and attosecond pulses in atomic gases. Opt. Express. 15, 15351-15364 (2007).

32. Ferrari, F. et al. High-energy isolated attosecond pulses generated by abovesaturation few-cycle fields. Nat. Photon. 4, 875-879 (2010).

33. Calegari, F. et al. Quantum path control in harmonic generation by temporal shaping of few-optical-cycle pulses in ionizing media. Phys. Rev. A 84, 041802(R) (2011).

34. Lucchini, M., Calegari, F., Kim, K. S., Sansone, G. \& Nisoli, M. Nonadiabatic quantum path analysis of the high-order harmonic generation in a highly ionized medium. New J. Phys. 14, 033009 (2012).

35. Zhao, K. et al. Tailoring a 67 attosecond pulse through advantageous phase-mismatch. Opt. Lett. 37, 3891-3893 (2012).

\section{Acknowledgements}

We would like to thank Dr. H. Geiseler for careful reading. This research was supported by Grant-in-Aid for Scientific Research (S) Grant Number 23226003 and Grant-in-Aid for Young Scientists (B) Grant Number 25790063 by Japan Society for the Promotion of Science, and the Photon Frontier Network Program by Ministry of Education, Culture, Sports, Science and Technology (MEXT), Japan.

\section{Author contributions}

N.I. conceived the experiment. N.I., T.K., and J.I. built the light source. N.I. and K.Kaneshima performed the HHG measurements. N.I. carried out the simulations. N.I. and J.I. wrote the manuscript. J.I. supervised the whole experiment. All authors discussed the results and manuscript.

\section{Additional information}

Supplementary Information accompanies this paper at http://www.nature.com/ naturecommunications

Competing financial interests: The authors declare no competing financial interests.

Reprints and permission information is available online at http://npg.nature.com/ reprintsandpermissions/

How to cite this article: Ishii, N. et al. Carrier-envelope phase-dependent high harmonic generation in the water window using few-cycle infrared pulses. Nat. Commun. 5:3331 doi: $10.1038 /$ ncomms4331 (2014).

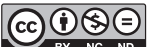

This work is licensed under a Creative Commons AttributionNonCommercial-NoDerivs 3.0 Unported License. To view a copy of this license, visit http://creativecommons.org/licenses/by-nc-nd/3.0/ 